\title{
Carnitine Palmitoyltransferase 1b Deficiency Protects Mice from Diet- Induced Insulin Resistance
}

Teayoun Kim¹, Lan He'1, Maria S. Johnson'1, Yan Li', Ling Zeng',2, Yishu Ding ${ }^{1}$, Qinqiang Long', John F. Moore ${ }^{3}$, Jon D. Sharer ${ }^{3}$, Tim R. Nagy', Martin E. Young $^{4}$, Philip A. Wood ${ }^{5}$ and Qinglin Yang ${ }^{1 *}$

${ }^{1}$ Department of Nutrition Sciences, University of Alabama at Birmingham, USA

${ }^{2}$ Department of Anatomy, Guangzhou University of Chinese Medicine, Higher Education Mega Center Campus, China

${ }^{3}$ Department of Genetics, University of Alabama at Birmingham, USA

${ }^{4}$ Department of Medicine, University of Alabama at Birmingham, USA

${ }^{5}$ Sanford-Burnham Medical Research Institute at Lake Nona, Orlando, USA

\begin{abstract}
Background: Carnitine Palmitoyl Transferase 1 (CPT1) is the rate-limiting enzyme governing long-chain fatty acid entry into mitochondria. CPT1 inhibitors have been developed and exhibited beneficial effects against type II diabetes in short-term preclinical animal studies. However, the long-term effects of treatment remain unclear and potential non-specific effects of these CPT1 inhibitors hamper in-depth understanding of the potential molecular mechanisms involved.
\end{abstract}

Methods: We investigated the effects of restricting the activity of the muscle isoform CPT1b in mice using heterozygous CPT1b deficient $\left(C p t 1 b^{+/}\right)$and Wild Type (WT) mice fed with a High Fat Diet (HFD) for 22 weeks. Insulin sensitivity was assessed using Glucose Tolerance Test (GTT), insulin tolerance test and hyperinsulinemic euglycemic clamps. We also examined body weight/composition, tissue and systemic metabolism/energetic status, lipid profile, transcript analysis, and changes in insulin signaling pathways.

Results: We found that $C p t 1 b^{+/-}$mice were protected from HFD-induced insulin resistance compared to WT littermates. Cpt $1 b^{+/}$mice exhibited elevated whole body glucose disposal rate and skeletal muscle glucose uptake. Furthermore, $\mathrm{Cpt}_{1 \mathrm{~b}^{+/}}$skeletal muscle showed diminished ex vivo palmitate oxidative capacity by $\sim 40 \%$ and augmented glucose oxidation capacity by $\sim 50 \%$ without overt change in whole body energy metabolism. HFD feeding $\mathrm{Cpt} \mathrm{b}^{+/-}$but not WT mice exhibited well-maintained insulin signaling in skeletal muscle, heart, and liver.

Conclusion: The present study on a genetic model of CPT1b restriction supports the concept that partial CPT1b inhibition is a potential therapeutic strategy.

Keywords: CPT1b; Fatty acid oxidation; Insulin sensitivity; Skeletal muscle

Abbreviations: PKB: Protein Kinase B; CHD: Chow Diet; MAPK1: Mitogen-Activated Protein Kinase 1; FAO: Fatty Acid Oxidation; HFD: High Fat Diet; IRS1: Insulin Receptor Substrate 1; ITT: Insulin Tolerance Test; OGTT: Oral Glucose Tolerance Test; WT: Wild Type

\section{Introduction}

Increased fatty acid availability and the concomitantly augmented mitochondrial fatty acid oxidation (FAO) capacity in skeletal muscle is a common feature in insulin resistant subjects $[1,2]$. Interfering with excessive fatty acid availability and oxidation hence may be a potential therapeutic strategy. Accumulated evidence has emerged that inhibiting FAO may sensitize insulin signaling in animals with insulin resistance [3-7] and in diabetic patients [7-9]. CarnitinePalmitoylTransferase 1 (CPT1) on the mitochondrial outer membrane is a rate limiting enzyme that converts long-chain acyl CoA into long chain acylcarnitine, thus being one of the most studied therapeutic targets for FAO inhibition.

There are three isoforms of CPT1 ( $a, b$ and $c)$. While CPT1a is expressed ubiquitously but most abundantly in the liver, CPT1b is expressed in skeletal muscle, heart, and adipose tissues, and CPT1c is expressed in the brain and testes (reviewed in [10]). HFD-fed mice show increased capacity of fatty acid oxidation in skeletal muscle homogenate and mitochondrial respiration with concomitantly increased CPT1 activity [11]. The expression of CPT1bis inversely related to insulin sensitivity among quartiles of metabolic syndrome patients [12]. Repressing the capacity of fatty acid oxidation may ameliorate insulin resistance. In supporting this hypothesis, several groups reported that CPT1 inhibitors improve insulin resistance in relatively short-term preclinical animal studies [5-8,13,14], even with increased intracellular lipid content. CPT1 inhibition in molecular genetic models due to the deletion of Malonyl-CoADecarboxylase (MCD) shows similar effects. The MCD null $(\mathrm{Mcd}$ /) mice are protected from HFD-induced insulin resistance by substantially increased Malonyl-CoA-Mediated repression of CPT1, consequently decreasing fatty acid oxidation and increasing glucose oxidation [3]. On the other hand, a $20 \%$ electroporation-mediated transient upregulation of CPT1b in the distal hind limb muscles is sufficient to improve HFD-induced insulin resistance in rats via attenuating triacylglycerol content, the membrane-to-cytosolic ratio of diacylglycerol, and protein kinase C $\theta$ activation [15]. Therefore, further studies on thein vivo roles of specific CPT1 isoform using the more specific gene manipulating approach are essential to resolve

*Corresponding author: Qinglin Yang, University of Alabama at Birmingham Nutrition Sciences, 1675 University Blvd, Webb 434, Birmingham, AL 35294, USA, Tel: 1-205-934-6023; E-mail: qyang@uab.edu

Received March 16, 2014; Accepted April 20, 2014; Published April 25, 2014

Citation: Kim T, He L, Johnson MS, Li Y, Zeng L, et al. (2014) Carnitine Palmitoyltransferase 1b Deficiency Protects Mice from Diet-Induced Insulin Resistance. J Diabetes Metab 5: 361 doi:10.4172/2155-6156.1000361

Copyright: @ $2014 \mathrm{Kim} \mathrm{T}$, et al. This is an open-access article distributed under the terms of the Creative Commons Attribution License, which permits unrestricted use, distribution, and reproduction in any medium, provided the original author and source are credited. 
these seemingly contradictory observations. The goal of the present study is to elucidate the effects of restricting fatty acid oxidation on diet-induced insulin resistancein the heterozygous Cpt1b knockout mice. This is the first study using a preclinical mouse model with Cpt1b-specific knockdown to investigate the long-term effects of CPT1b repression on HFD-induced insulin resistance and the underlying metabolic mechanisms.

\section{Materials and Methods}

\section{Animals}

The heterozygous Cpt1 $1 b^{+/-}$knockout mice [16] and wild-type (WT) littermates in C57BL/6J background were used. While the homozygous CPT1b knockout is lethal, $\mathrm{Cpt}_{1 b^{+/-}}$mice show no overt abnormal phenotype and whole body metabolic changes [16]. All mice were kept on 12-hour/12-hour light/dark cycle (light on at 06:00 hours) at 22.0 $\pm 1.0^{\circ} \mathrm{C}$ and had $a d$ libitum access to water and standard rodent diet (Harlan Laboratories 7017 NIH-31 Mouse/Rat Sterilizable Diet, 14\% $\mathrm{kcal} \%$ fat). Mice (4 - 5 week old, male) of high fat diet (HFD) feeding groups were given ad libitumaccess to HFD (60\% kcal\% fat) (Research DIETS D12492) and water. Body weights were recorded every other week. All experimental procedures were conducted in accordance with the Guide for Care and Use of Laboratory Animals and were approved by the Institutional Animal Care and Use Committee of the University of Alabama at Birmingham (UAB).

\section{Oral Glucose Tolerance Test (OGTT) and Insulin Tolerance Test (ITT)}

Mice were fasted overnight for OGTT (18:00 - 08:00) or 5 hours for ITT (08:00-13:00). Blood glucose levels were measured on blood samples from tail tip-snip using Contour glucometer (Bayer) after glucose administration (gavage $1.5 \mathrm{mg} / \mathrm{kg}$ b.w.) or insulin injection (Humulin R, Eli Lilly \& Co, i.p. $0.5 \mathrm{U} / \mathrm{kg}$ b.w.).

\section{Hyperinsulinemiceuglycemic clamp}

The procedures of hyperinsulinemiceuglycemic clamp (insulin clamp) in mice were adapted from Dr. Pessin'sgroup[17] with minor modifications based on reports from other groups [18,19]. Briefly, mice were anesthetized by Isoflurane via a Vaporizer-MiniVentmouse ventilator system (HUGO SACHS ELECTRONIK, Harvard Apparatus $\mathrm{GmbH}$, Hugstetten, Germany). A catheter was surgically implanted into the right jugular vein and threaded under the dorsal skin of mice. Three days after surgery, the mouse was fasted 5 hrs (08:00-13:00) and then placed in a rat-size restrainer with its tail taped. The catheter was connected to a CMA 402 syringe pump (CMA Microdialysis, Stockholm, Sweden). $\left[6{ }^{3} \mathrm{H}\right]$-glucose was infused at $0.5 \mu \mathrm{Ci} / \mathrm{min}$ for $2 \mathrm{hrs}$ without insulin and then infused at $1 \mu \mathrm{Ci} / \mathrm{min}$ with insulin (Humulin R, Eli Lilly $2.5 \mathrm{mU} \mathrm{kg}^{-1} \mathrm{~min}^{-1}$ ) for $2 \mathrm{hrs}$, by which time the blood glucose was maintained at $7.8-8.9 \mathrm{mmol} / \mathrm{L}$ by adjusting $20 \%$ glucose infusion rate in the mouse under the conscious condition. 10 $\mu \mathrm{Ci} 2$ - $\left[{ }^{14} \mathrm{C}\right]$-deoxy-D-glucose was infused $40 \mathrm{~min}$ before the end of the 120 min euglycemic clamp. The blood glucose level was measured from tail tip snipped blood samples using a Contour glucometer (Bayer). At the end of the clamp study, tissues were harvested and snapped frozen in liquid nitrogen after the mouse was euthanized. The plasma glucose level was measured using an Analox GM7 Micro-Stat Analyzer (Analox Instruments, London, UK). The specific activity of plasma glucose, the glucose infusion rate (GIR), the whole body glucose disposal rate (Gd), and the tissue-specific glucose uptake were measured and calculated as previously described [20].

\section{Serum analysis}

Tissues and sera were collected from sacrificed mice after overnight fasting (18:00 - 08:00). Insulinlevelwas measured using a RIA kits (Millipore Co. SRI-13K, ML-82K). The content of Non-Esterified Fatty Acids (NEFA) was measured using a NEFA-HR Kit (Wako), respectively.

\section{Lipid measurements}

Frozen gastrocnemius muscles were pulverized using a pulverizor (Bio Spec Products Inc.) in liquid nitrogen and weighed in small tubes as previously described [21]. For the non-esterified fatty acids (NEFA), lipids were extracted using the Bligh \& Dyer method [22].NEFA and TAG were measured using a NEFA-HR Kit (Wako) and a Triglyceride Quantification Kit (BioVision K622-100). For the acylcarnitine assay, 6 volume of $80 \%$ acetonitrile was added to pulverized tissue weight (about $50 \mathrm{mg}$ ). Tissue mixtures were sonicated 10 times, centrifuged at $12,000 \mathrm{rpm} 10 \mathrm{~min}$ at $4{ }^{\circ} \mathrm{C}$, and supernatants were transferred to new tubes. The supernatants were dried under a stream of nitrogen at $40{ }^{\circ} \mathrm{C}$ and resuspended in $100 \mu \mathrm{l}$ of $50 \%$ acetonitrile. The acylcarnitine content was measured by using Electrospray Ionization Tandem Mass Spectrometry [23].

\section{Ex vivo oxidation assay}

Intact muscle oxidation assay was performed as previously described [24]. Extensor digitorumlongus (EDL) muscles were excised from euthanized mice and incubated with $700 \mu \mathrm{l}$ of Krebs-Ringer Phosphate buffer containing $0.1 \mu \mathrm{Ci} / \mathrm{ml}$ of BSA-conjugated $\left[{ }^{14} \mathrm{C}\right]-$ palmitateor $\left[{ }^{14} \mathrm{C}\right]$-glucose in sealed $14 \mathrm{ml}$ tubes with center wells containing $1 \mathrm{~N} \mathrm{NaOH}$ at $37^{\circ} \mathrm{C}$ for 1 hour with $200 \mathrm{rpm}$ shaking. After incubation, $400 \mu \mathrm{l}$ of $3.5 \mathrm{M} \mathrm{HClO}_{4}$ was injected into the media and incubated at $50^{\circ} \mathrm{C}$ for 3 hours to capture oxidized substrates to $\mathrm{NaOH}$ and the radioactivity was measured by scintillation counter [24].

\section{Oxidation assays in isolated mitochondria}

The procedures of mitochondrial oxidation assay were adapted from Dr. Kove's group as previously described [3]. Gastrocnemius muscle (one hind limb from one mouse) was homogenized by using hand-held drill with Potter-Elvehjem homogenizer. Supernatants were kept following a low speed $(1,000 \mathrm{xg})$ centrifugation, and subsequently centrifuged again at 12,000 $\mathrm{xg}$ to isolate mitochondria. Mitochondrial protein concentration was measured by Lowry method and $100 \mu \mathrm{g}$ of mitochondria was incubated with $0.1 \mu \mathrm{Ci} / \mathrm{ml}$ of $\left[{ }^{14} \mathrm{C}\right]$-palmitate including reaction buffer for $2 \mathrm{hrs}$. The oxidation reaction was stopped by injecting perchloric acid. Oxidized substrates were metabolized as ${ }^{14} \mathrm{CO}_{2}$ and captured by $\mathrm{NaOH}$ in the central well and the radioactivity was measured by scintillation counter. Incompletely oxidized substrates (acid soluble metabolites-ASM) remained in the reaction mixture were centrifuged. Then the supernatants were used to detect ${ }^{14} \mathrm{C}$ by liquid scintillation counter.

\section{Real-time qPCR}

Total RNA extraction and cDNA synthesis were done by using RNeasy` Mini kit (QIAGEN), Advantage RT-for PCR kit (Clontech), Power SYBR' Green PCR master mix. The real-time PCR was performed by using Step One Real-Time PCR System (Applied Biosystems) with standard curve for the transcript quantification. The primer sequences are listed in Supplementary Table 1.

\section{Western blot analysis}

Frozen gastrocnemius muscles, liver, and hearts were homogenized 


\begin{tabular}{|c|c|c|c|c|c|c|}
\hline \multirow{2}{*}{$\begin{array}{l}\text { Time } \\
\text { strain }\end{array}$} & \multicolumn{2}{|c|}{8 weeks of HFD } & \multicolumn{2}{|c|}{22 weeks of HFD } & \multicolumn{2}{|c|}{22 weeks of HFD Clamp } \\
\hline & WT & CPT1b ${ }^{+/-}$ & WT & CPT1b ${ }^{+/-}$ & WT & CPT1b ${ }^{+/-}$ \\
\hline Glucose (mg/dl) & $82.7 \pm 3.3$ & $73.1 \pm 4^{*}$ & $148 \pm 6.3^{\ddagger \ddagger}$ & $109 \pm 11^{\neq \ddagger} *$ & $162 \pm 28$ & $158 \pm 25$ \\
\hline $\mathrm{NEFA}(\mathrm{mEq} / \mathrm{L})$ & $1.3 \pm 0.2$ & $1.0 \pm 0.2$ & $1.1 \pm 0.1$ & $1.3 \pm 0.2$ & $0.9 \pm 0.14$ & $0.5 \pm 0.06^{*}$ \\
\hline Insulin $(\mu \mathrm{lU} / \mathrm{ml})$ & $6.3 \pm 0.4$ & $6.3 \pm 0.4$ & $10.7 \pm 0.9^{\ddagger \ddagger}$ & $8.3 \pm 0.6^{{ }^{*}}$ & $37.5 \pm 7.5$ & $45 \pm 4.9$ \\
\hline Leptin $(\mu \mathrm{g} / \mathrm{L})$ & $5.0 \pm 2$ & $27.1 \pm 21$ & $35.4 \pm 11^{\ddagger}$ & $19.4 \pm 4.0$ & ND & ND \\
\hline
\end{tabular}

Mice were fasted for overnight. $n=4-7,{ }^{*} p<0.05,{ }^{* *} p<0.01$ between WT and CPT1 $b^{+/-}$mice within the same time point, ${ }^{\ddagger} p<0.05$, ${ }^{\ddagger \pm} p<0.01$ between 8 and 22 weeks within the same group.

Table 1: Plasma lipid and hormone profile after 8 weeks, 22 weeks of HFD feeding, and insulin clamp (n=7-8).

using a pestle pellet mini homogenizer in homogenization buffer (50 $\mathrm{mM}$ TrisHCl pH 6.8, 1\% SDS, $2.5 \mathrm{mM}$ DTT, 10\% glycerol). The protein concentration of supernatant was measured by using Modified Lowry Protein Assay Kit (Pierce \#23240). Primary antibodies were purchased from Cell Signaling; pIRS1 Ser302 (\#2491), IRS1 (\#2390), pAKT Ser473 (\#9271), AKT (\#9272), pERK1/2 (p44/42 MAPK) Thr202/Tyr204 (\#9101), and ERK1/2 (\#9102). HRP-conjugated secondary antibodies were from Santa Cruz Biotechnology. Western blot images were taken and quantified by using Kodak Image Station 4000R (Molecular Imaging System, Carestream Health Inc., Rochester, NY, USA).

\section{Body composition analysis}

Fat and lean mass were measured in vivo using a quantitative magnetic resonance imaging system (QMR, EchoMRI ${ }^{\mathrm{TM}}$ 3-in-1, Echo Medical System, Houston, TX, USA) at UAB Small Animal Physiology Core as previously described and validated [25].

\section{Comprehensive Lab Animal Monitoring System (CLAMS) analysis}

Respiration rate, food intake, energy expenditure, and physical activity were accurately quantified as previously described using the CLAMS (Columbus Instruments Inc., OH, USA) [26]. Mice were individually kept in CLAMS chambers with ad libitum access to food and water for 6 days and the data sets for the last 3 days were averaged for the analysis. Respiratory exchange ratio (RER) was calculated as $\mathrm{CO}_{2}$ generation $/ \mathrm{O}_{2}$ consumption. Energy expenditure was expressed as (kcal/hr).

\section{Indirect calorimetry analysis}

Energy expenditure, RER, and activity were also measured with another set of mice using an 8-cage indirect calorimetry system (CaloSys, TSE Systems, Bad Homburg, Germany) as previously described [27]. Mice were individually kept in airtight plastic cages with ad libitum access to food and water and a continuous flow of air was maintained through all cages. Mice were acclimated to the cages for 48 hours prior to the measurement period of 22 hours. Total energy expenditure was calculated over the 22 hours, and presented as per 24-hours. Resting energy expenditure was calculated as the average of the 3 lowest 18 minute periods during the measurement.

\section{Rectal temperature measurement}

Body temperatures of mice were measured using rectal probe provided from Echocardiogram (Vevo770, Visualsonics Inc., Toronto, Ontario, Canada) under isoflurane-induced anesthesia at around 13:00.

\section{Statistical analysis}

GraphPad Prism5 software was used to conduct Two-tailed Student's $t$-test and One-Way ANOVA with Tukey post hoc test. The ANCOVA was done using SAS9.1 software. Differences between groups were regarded significant at $\mathrm{p}<0.05$ probability level. Data are expressed as means $\pm \mathrm{SE}$.

\section{Results}

Mice with CPT1b deficiency are protected against HFDinduced insulin resistance

We investigated whether partial CPT1b deficiency determines insulin sensitivity in mice under CHD and HFD feeding conditions using OGTT and ITT. At baseline (CHD 4 weeks), CHD-fed Cpt1 $b^{+\prime}$ and WT littermates showed no difference in OGTT and ITT (Figure $1 \mathrm{~A}$ and 1B). After 8 weeks of HFD feeding, Cpt $1 b^{+/-}$mice showed attenuated blood glucose levels at 60 minutes time point during OGTT (Figure 1C). The blood glucose levels during ITT were not changed at the 30 minute time point, but were lower in $C p t 1 b^{+/-}$than in WT mice at 60 and 120 minute time points (Figure 1D). At 22 weeks after HFD feeding, $C p t 1 b^{+/-}$mice showed substantially improved glucose tolerance compared with WT littermates. The area under curve (AUC) of OGTT in $C p t 1 b^{+/-}$mice was decreased about $45 \%$ compared with WT (Figure 1E). The blood glucose levels during ITT were not changed at the 30 minute time point but lower in $\mathrm{Cpt}_{1 \mathrm{~b}^{+/-}}$than in WT mice at the later time points (Figure 1F). These results indicate that CPT1b deficiency improves glucose and insulin tolerance under a HFD condition. Cpt $1 b^{+1-}$ mice showed no difference in ITT and OGTT until 32 weeks of their age (Supplemental Figure 1) suggesting that CPT1b deficiency does not alter insulin sensitivity and glucose tolerance under a normal diet condition.

To gain insights into the tissue-specific insulin sensitivities, we further assessed HFD feeding mice using the hyperinsulinemiceuglycemic clamp at 22 weeks after HFD feeding. A much higher glucose infusion rate (GIR) in $\mathrm{Cpt} 1 \mathrm{~b}^{+/-}$mice than in WT mice was required to maintain blood glucose levels $(7.8-8.9 \mathrm{mmol} / \mathrm{L})$ in response to the constant insulin infusion (Figure 1G). Consistently, whole body glucose disposal rate $(\mathrm{Gd})$ was higher in Cpt $1 b^{+/-}$than in WT mice (Figure $1 \mathrm{H}$ ), indicating improved systemic insulin sensitivity in the $C p t 1 b^{+/-m i c e}$ compared with WT mice. Insulin-stimulated glucose uptake was increased by about 2-fold in $\mathrm{Cpt}_{1 b^{+/-}}$relative to WT skeletal muscle (Figure 1), whereas there was no difference between two groups in Gonadal White Adipose Tissue (GWAT)(Figure 1H). The plasma glucose level was significantly lower in $\mathrm{Cpt} 1 \mathrm{~b}^{+/-}$mice than in WT littermates at 8 weeks and 22 weeks of HFD feeding. No changes could be detected in plasma NEFA throughout the observation period in both groups. On the other hand, plasma insulin $(\mathrm{p}<0.01)$ and leptin levels surged after 22 weeks of HFD in WT from 8 weeks, while insulin levels in $C p t 1 b^{+/-}$mice were substantially lower than their WT controls at 22 weeks. The plasma insulin level during insulin clamp was not different between two groups, while plasma NEFA level was substantially decreased in $\mathrm{Cpt}_{1} b^{+-}$mice (Table 1).

CPT1b deficiency in mice leads to repressed fatty acid oxidation and enhanced glucose oxidation in the skeletal muscle under a HFD condition

We further investigated whether changes in substrate metabolism 
A

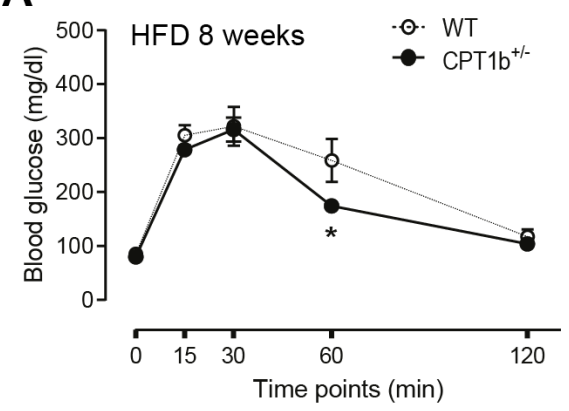

C

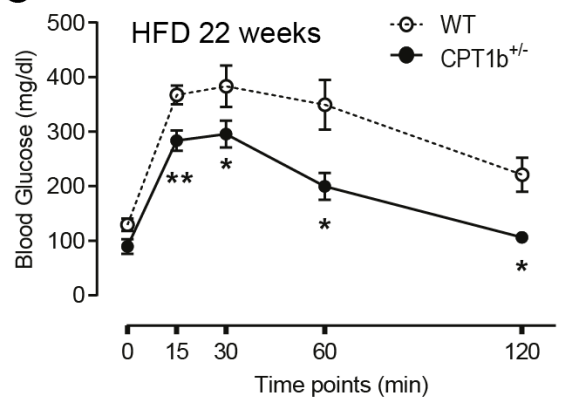

B
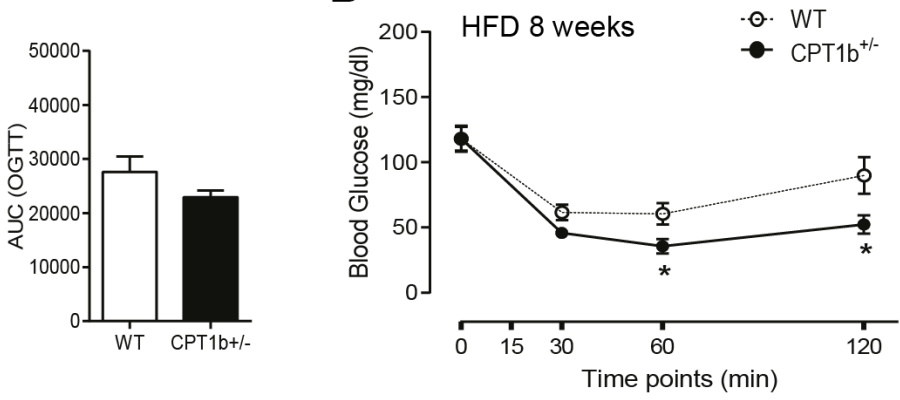

D

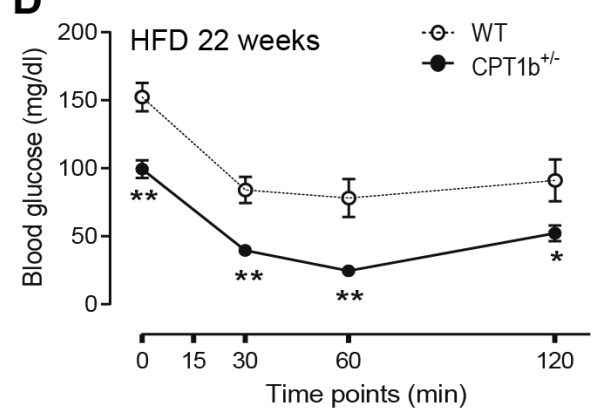

$\mathbf{E}$

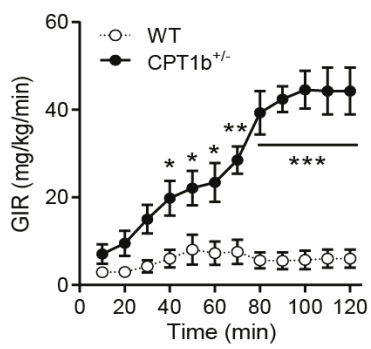

$\mathbf{F}$

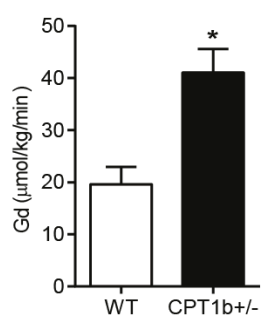

G

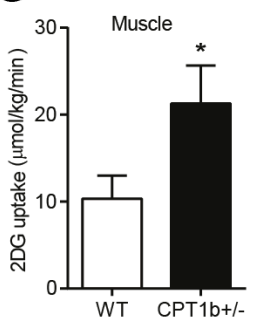

H

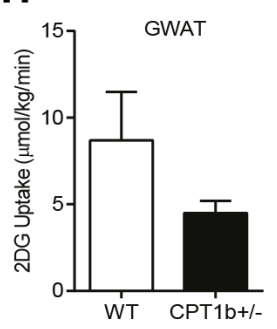

Figure 1: $C p t 1 b^{+/-}$mice are insulin sensitive under a HFD condition

Insulin sensitivity was assessed by OGTT, ITT, and Insulin clamp study at different time points. (A) OGTT in mice with 8 weeks of HFD. (B) ITT in mice with 8 weeks of HFD. (C) OGTT in mice with 22 weeks of HFD. (D) ITT in mice with 22 weeks of HFD. Bar graphs are the area under curve (AUC) of OGTT calculated from the original graph. (E) Glucose infusion rate (GIR) during last 40 minutes of insulin-stimulated condition in mice with 22 weeks of HFD. (F) Whole body glucose disposal rate (Gd) in mice with 22 weeks of HFD. (G) Glucose uptake into gastrocnemius muscle in mice with 22 weeks of HFD. (H) Glucose uptake into GWAT in mice with 22 weeks of HFD. $n=4-6,{ }^{*} p<0.05,{ }^{* *} p<0.01,{ }^{* * \star} p<0.001$.

correlated with different insulin sensitivity in $C p t 1 b^{+/-}$vs. WT mice. CPT1b deficient muscle did not show decreased palmitate oxidation capacity compared to WT muscle under a CHD condition (Figure 2A). The rate of ex vivo palmitate oxidation in skeletal muscle of $\mathrm{Cpt1b^{+/- }}$ mice after 22 weeks of HFD feeding was $40 \%$ lower than in WT mice $(\mathrm{p}<0.05)$ (Figure $2 \mathrm{~A})$, whereas the rate of ex vivo glucose oxidation was $50 \%$ higher in $\mathrm{Cpt}_{1} \mathrm{~b}^{+/-}$than in WT muscles (Figure 2B). Other tissues such as heart, brown adipose tissue, and liver showed no difference in the ex vivo oxidation capacity between two groups (data not shown) under a HFD condition. The palmitate oxidation rate in isolated mitochondria was also decreased in $\mathrm{CPT} \mathrm{b}^{+/}$compared with WT muscle (Figure 2C) with no change in incomplete oxidation (Figure 2D). To assess whether the decreased fatty acid oxidation capacity would result in intramyocellular lipid accumulation, skeletal muscle lipid contents were assessed after 22 weeks of HFD feeding. There was no difference in TAG and NEFA levels between $C p t 1 b^{+/-}$and WT muscle (Figure 2E and 2F). Notably, acylcarnitine profile in skeletal muscle of HFD-feeding Cpt $1 b^{+/-}$mice was also not different from WT (Figure 2G). In the transcript analysis, Cpt2 ( $\mathrm{p}<0.01), C p t 1 a, A c s$ (acyl-CoA synthase long-chain family member 1), Dgat (diacylglycerol O-acyltransferase 2), Gpam (glycerol-3-phosphate acyltransferase, mitochondrial), and Mlycd (malonyl-CoA decarboxylase) were significantly decreased in $C p t 1 b+/-$ muscle $(\mathrm{P}<0.05)$ (Supplemental Figure 2) supporting that lipid was not accumulated in $C p t 1 b+/-$ muscle. Therefore, the above results implicate that modest repression of FAO in skeletal muscle could increase glucose oxidation without overt intramuscular lipid accumulation.

\section{Insulin signal transduction is well maintained in $C_{p t 1 b^{+/}}$ mice skeletal muscles}

To confirm the improved insulin sensitivity of $C p t 1 b^{+/-}$mice under a long-term HFD condition on insulin signaling, we subjected tissue samples from hyperinsulinemiceuglycemic clamp study to Western blot analysis of key insulin signaling proteins. The phosphorylation of IRS1 at Ser302 was substantially lower in $C p t 1 b^{+/-}$than in WT muscles $(\mathrm{p}<0.01)$ (Figure 3A), but there was no difference in the heart (Figure 3B). No signals of phosphorylation of IRS1 at Ser302 were 

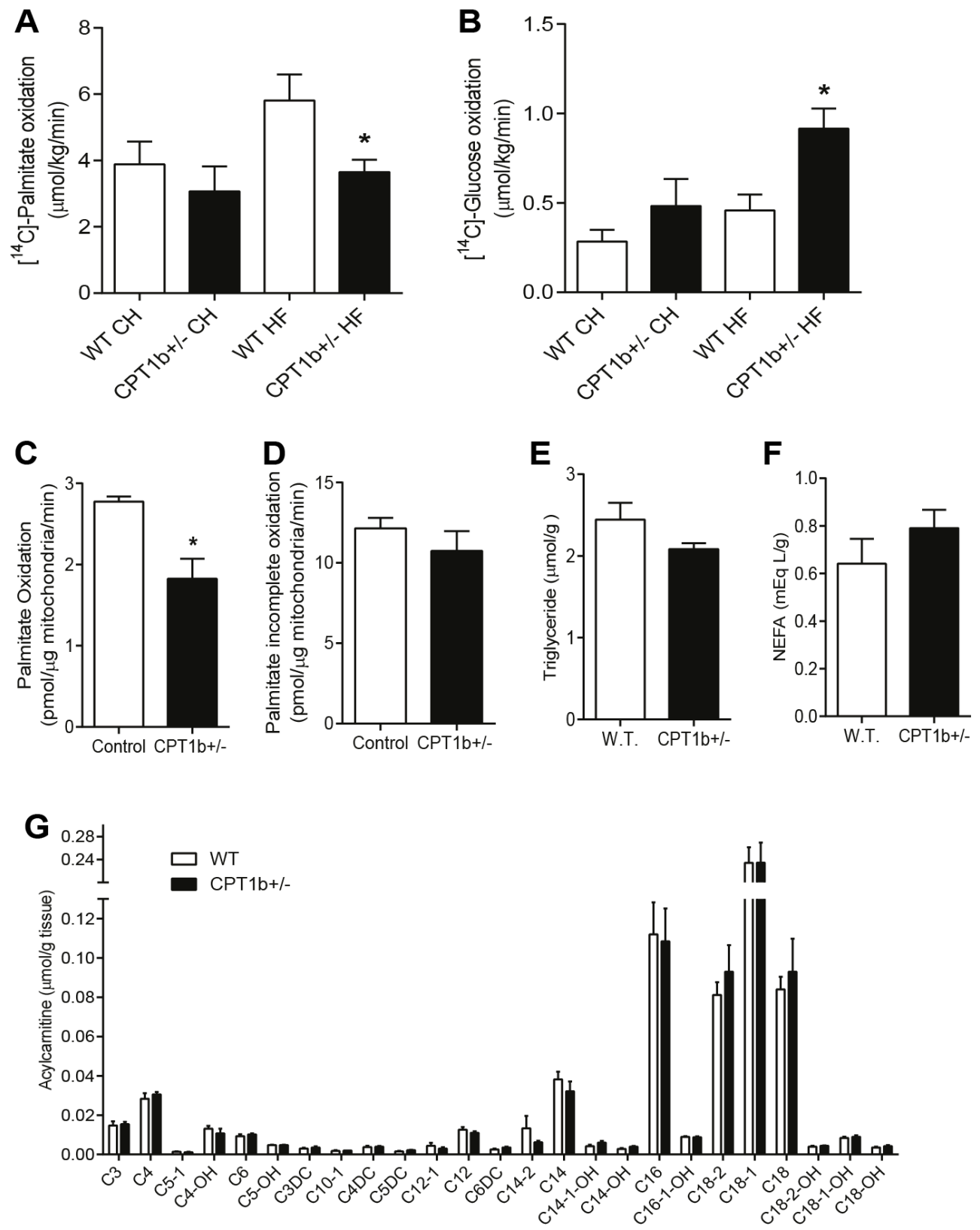

Figure 2: CPT1b deficiency leads to suppressed fatty acid oxidation and increased glucose oxidation without lipid accumulation in the skeletal muscle Substrate oxidation rate and lipid contents in the skeletal muscle of mice were assessed after 22 weeks of HFD feeding. (A) Ex vivo [ $\left.{ }^{14} \mathrm{C}\right]-$ palmitate oxidation in EDL muscle. (B) Ex vivo $\left[{ }^{14} \mathrm{C}\right]$-D-glucose oxidation in EDL muscle. (C) $\left[{ }^{14} \mathrm{C}\right]$-palmitate complete oxidation in isolated mitochondria from gastrocnemius muscle. (D) $\left[{ }^{14} \mathrm{C}\right]-$ palmitate incomplete oxidation in isolated mitochondria from gastrocnemius muscle. (E) Triglyceride content in gastrocnemius muscle. (F) Non-esterified free fatty acid (NEFA) content in gastrocnemius muscle. (G) Acylcarnitine content in gastrocnemius muscle. $n=5-6,{ }^{*} p<0.05,{ }^{* *} p<0.01,{ }^{* * *} p<0.001$.

detected in liver and GWAT (data not shown). The phosphorylation of AKT at Ser473 was substantially elevated in muscle $(\mathrm{p}<0.01)$ (Figure $3 \mathrm{~A})$, heart $(\mathrm{p}<0.01)$ (Figure $3 \mathrm{~B})$, and liver $(\mathrm{p}<0.01)$ (Figure $3 \mathrm{C})$, but decreased in GWAT (Figure 3D) $(\mathrm{p}<0.05)$ of Cpt1 $\mathrm{b}^{+/}$mice. The phosphorylation of ERK1/2 at Thr202/Tyr204 was maintained in muscle $(\mathrm{p}<0.05)$ (Figure $3 \mathrm{~A})$, liver $(\mathrm{p}<0.01)$ (Figure $3 \mathrm{C})$, but had no difference in heart (Figure $3 \mathrm{~B}$ ) and decreased in GWAT (Figure 3D) of $C p t 1 b^{+/-}$mice. These results demonstrate that $C p t 1 b^{+/-}$mice are protected from HFD-induced impaired insulin signaling in muscle, heart, and liver, but not in GWAT.

\section{Mice with CPT1b deficiency are smaller than control mice subjected to HFD}

We found no difference in body weight and body composition between $\mathrm{Cpt}_{1 b^{+-}}$and WT mice under a CHD condition throughout life-time (data not shown). However, Cpt $1 b^{+/}$mice showed a smaller increase of body weight than WT mice after 4 weeks of HFD feeding (Figure 4A and 4B).At 8 weeks after HFD feeding, Cpt $1 b^{+/-}$mice exhibited $50 \%$ less body fat mass and $14 \%$ less lean mass than WT mice (Figure 4C). However, ANCOVA analysis revealed that these changes were proportional to the total body mass, suggesting that the improved 
A

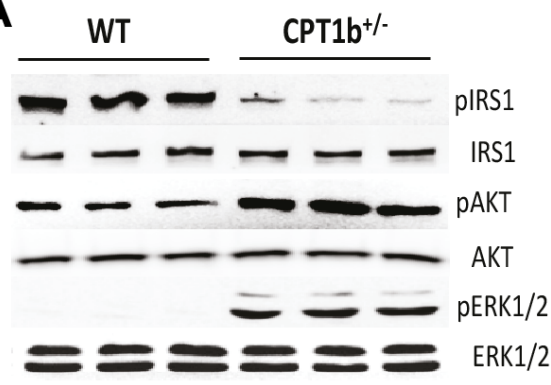

B

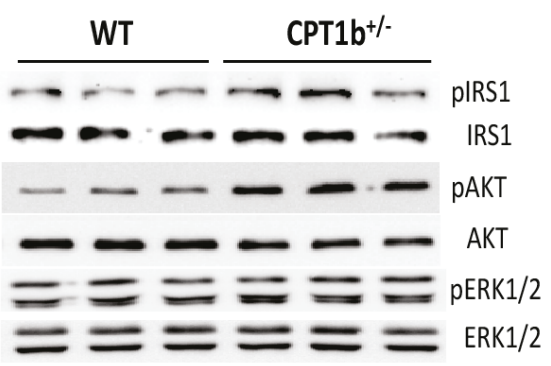

C

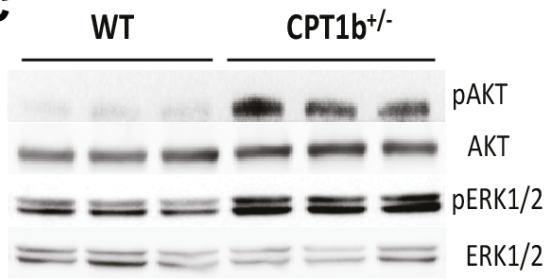

D

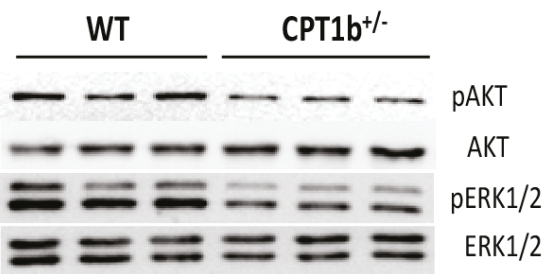

HFD-fed mice gastrocnemius muscle
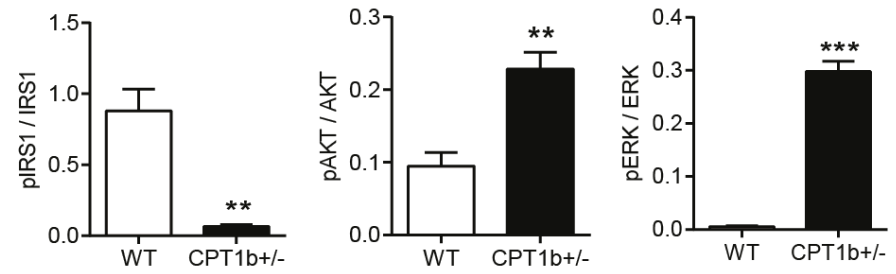

HFD-fed mice heart
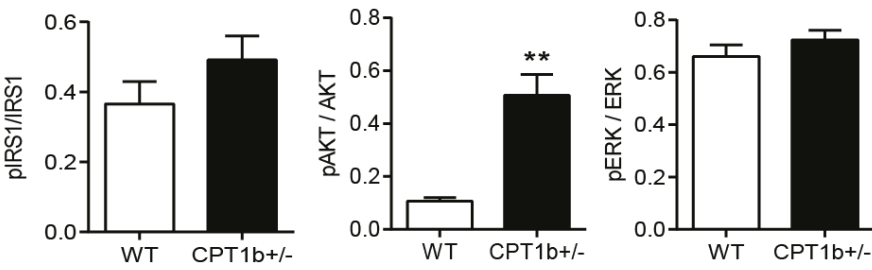

HFD-fed mice liver
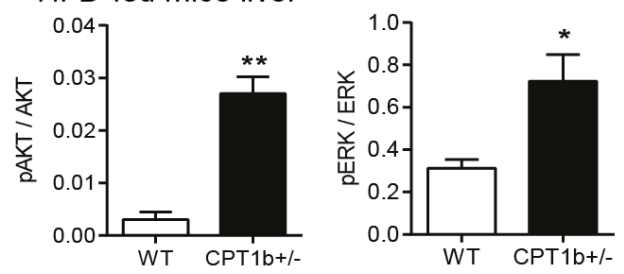

HFD-fed mice GWAT
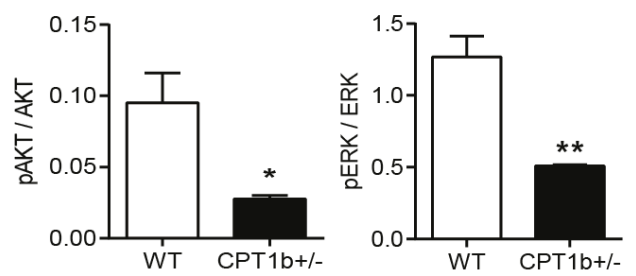

Figure 3: Insulin signaling is well maintained in the skeletal muscle of $C p t 1 b^{+/-}$mice

Tissue samples from Insulin clamp study after 22 weeks of HFD feeding were subjected to Western blot analysis for the phosphorylation of Ser 302 IRS1, Ser473 AKT, Thr 202/Tyr 204 ERK1/2 and total protein of each target. (A) Gastrocnemius muscle. (B) Heart. (C) Liver. (D) GWAT. $n=4,{ }^{*} p<0.05,{ }^{\star \star} p<0.01$.

insulin sensitivity of $\mathrm{Cpt}_{1 b^{+/-}}$mice is independent of changes in body fat mass.

\section{CPT1bdeficiency does not influence whole body energy balance in response to HFD feeding}

We next determined whether the altered substrate oxidation capacity of skeletal muscle in $\mathrm{Cpt}_{1} \mathrm{~b}^{+/-}$mice under a HFD condition affects the whole body energy balance. We measured respiratory exchange rate (RER), energy expenditure, activity, and food intake using CLAMS at 22 weeks after HFD feeding. Interestingly, none of these parameters were different between two groups (Figure 5A-5D). We confirmed the RER assessment of CLAMS analysis by using indirect calorimeter with another set of mice at 22 weeks after HFD feeding. $C p t 1 b^{+/-}$mice showed no difference in energy expenditure (Figure 5E) with very subtle decreased RER (Figure 5F), accompanied with weight loss compared to WT during the 2 day measurement period (Figure 5G). Consistently, the HFD-fed $\mathrm{WT}$, but not $\mathrm{Cpt} 1 \mathrm{~b}^{+/-}$mice, showed higher rectal temperature (Figure $5 \mathrm{H}$ ), suggesting that the whole body energy metabolism of $\mathrm{Cpt} 1 \mathrm{~b}^{+/-}$mice was not substantially altered. Analysis of fecal energy content revealed no differences between the two groups (data not shown). Therefore, reduced fatty acid oxidation and increased glucose oxidation in skeletal muscle appear to be in a balanced state that does not substantially alter whole body metabolism and energy balance in mice subjected to HFD.

\section{Discussion}

In the current study, we provide evidence supporting the beneficial 


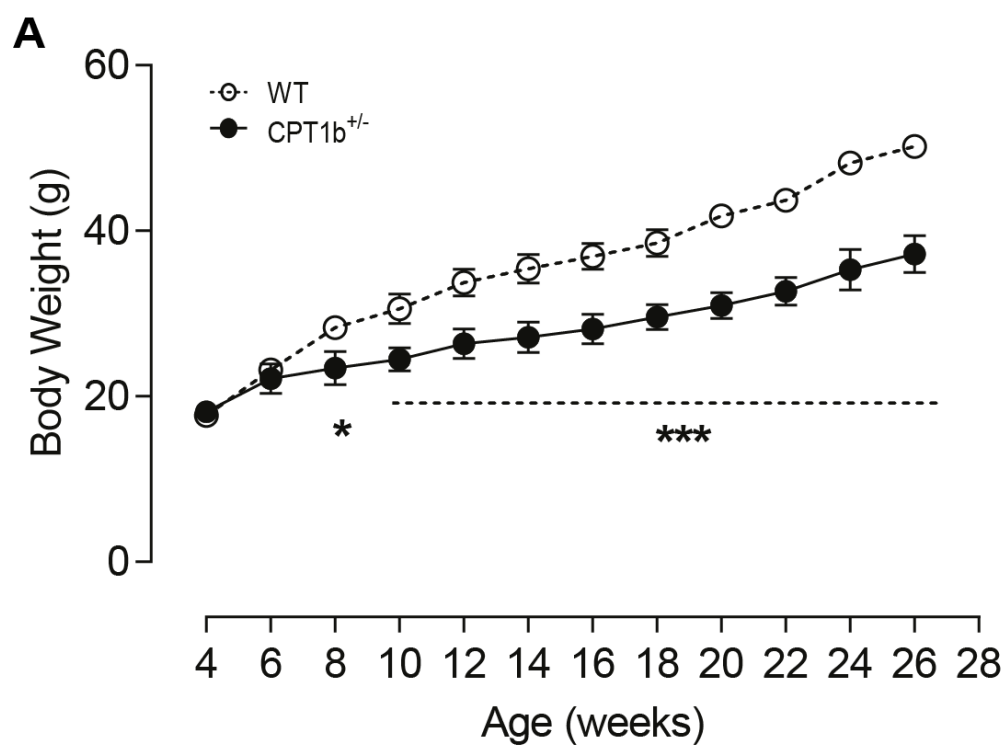

B

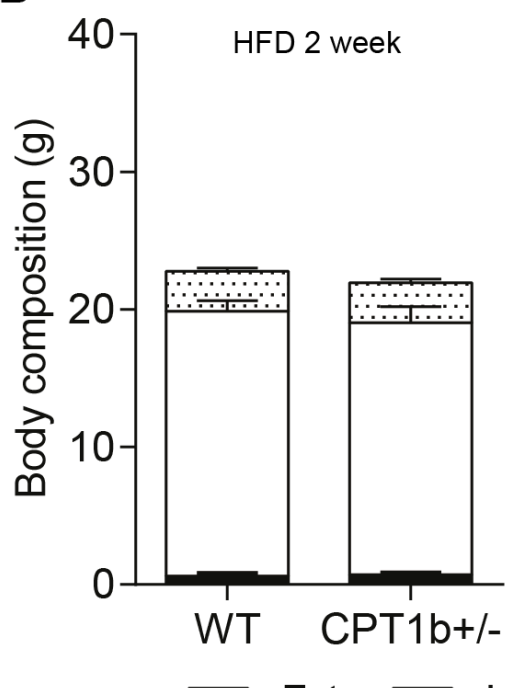

C

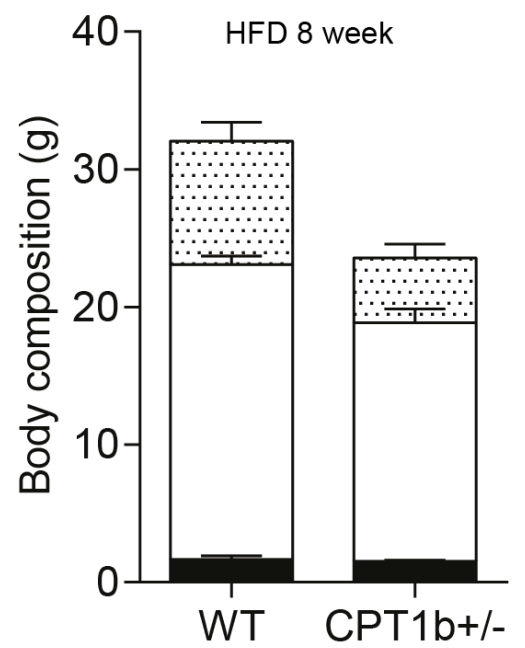

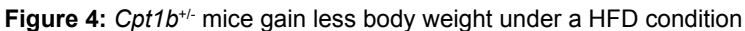

(A) Body weight trend in HFD. (B) Body composition (QMR) at 2 weeks of HFD feeding. (C) Body composition (QMR) after 8 weeks of HFD feeding. $n=6$, ${ }^{*} p<0.05$, ${ }^{* *} p<0.001$.

effects of CPT1b ablation related fatty acid oxidation restriction against insulin resistance induced by HFD. We investigated a genetic mouse model with $C p t 1 b^{+/-}$deficiency subjected to 22 weeks of HFD feeding. We illustrate that CPT1b mediated fatty acid oxidation in skeletal muscle is a crucial site for the maintenance of insulin sensitivity via adjustingthe balance of local fatty acid and glucose oxidation rates.

Pharmacological CPT1 inhibition has been suggested as an effective therapy to improve insulin sensitivity in type 2 diabetes patients for nearly two decades [28-30]. A CPT1 inhibitor, etomoxir, improves glucose homeostasis in patients by repressing fatty acid oxidation and elevating glucose oxidation, presumably by the Randle cycle mechanism, despite the subsequent intramyocellular lipid accumulation [7]. Nevertheless, opposite results have also been reported, in which etomoxir is associated with exacerbated insulin resistance with concomitantly increased intracellular lipid accumulation in rodents [31]. Oxfenicine, another CPT1 inhibitor, increases glucose oxidation in HFD-fed mice with increased respiratory exchange ratio (RER), but no intramyocellular lipid accumulation [4]. The inconsistent outcomes from the CPT1 inhibitor studies are most likely derived from the different experimental approaches and the different degrees/subtype specificities of these CPT1 inhibitors. While pharmacological studies provide highly clinical relevant insights into the effects of a specific CPT1 inhibitor, this approach does not target specific CPT1 isoforms with inherent non-CPT1 inhibition effects (e.g., etomoxir has been shown to be a PPARa activator [32]). Moreover, most of previous etomoxir studies were performed with high dosages and relatively shortterm assessments on the acute effects of etomoxir, which mostly acts 

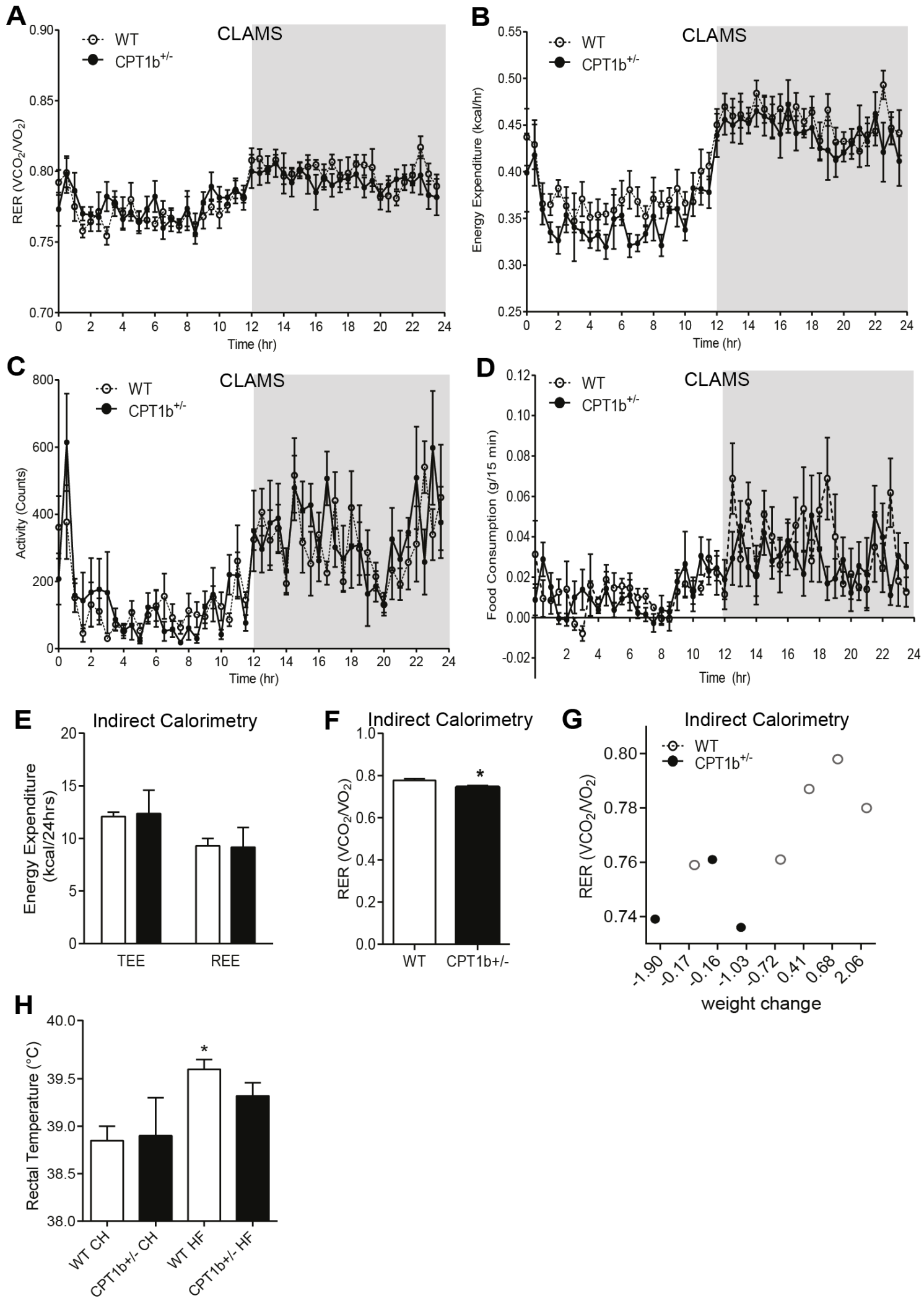

Figure 5: CPT1b deficiency does not alter whole body energy balance under a HFD condition

In vivo whole body metabolism was assessed at 22 weeks of HFD feeding using comprehensive Lab Animal Monitoring System (CLAMS) with the average of the last 3 days (A-D), $n=5-6$. (A) Respiratory exchange rate (RER). (B) Energy expenditure. (C) Activity. (D) Food intake. Indirect calorimeter analysis (E-G), $n=3-5$. (E) Energy expenditure. (F) Respiratory exchange rate (RER). (G) Correlation between body weights change and RER. (H) Rectal temperature. $n=4-5$, * $p<0.05$. 
on CPT1a, the liver isoform of CPT1 [33]. Oxfenicine, which inhibits more CPT1b than CPT1a [34], seems to be a promising anti-diabetic drug, since it improves insulin sensitivity without intramuscular lipid accumulation in mice [4]. It remains challenging to exclude the potential off-target effects in pharmacological studies. Additional studies on animal models with genetic manipulations should help overcome the related difficulties. Our current study using the genetic mouse model with CPT1b deficiency provides specific insights and clarifies the effect of CPT1b deficiency on HFD-induced insulin resistance.

Despite the encouraging support of CPT1b inhibition as a potential therapy for insulin resistance as reported here, many other studies using various CPT1 inhibitors have shown adverse effects [31,35] due to intramyocellular fatty acid-related metabolites (e.g., DAGs, acylcarnitines, ceramides) accumulation. In this study we prove that repressed fatty acid oxidation due to heterozygous CPT1b deficiency in skeletal muscle did not lead to accumulation of lipid metabolites, which is consistent with the Oxfenicine study [34]. Previous study showed increased fatty acid oxidation in ex vivo muscle tissues of HFD-fed WT mice [11]. Zucker diabetic fatty (ZDF) rats showed increased fatty acid oxidation-related gene expression in their muscle with increased muscle acylcarnitine accumulation [3]. In contrast, a human study showed that palmitate oxidation was significantly decreased, but only in extremely obese patients [36]. Additional studies also demonstrated opposite results either with reduced or elevated fatty acid oxidation in obese human skeletal muscle [37,38]. However, the current study did not detect substantial change at least in the EDL muscle before and after HFD. These different results may be derived from many factors, such as the degrees of obesity and the measurement methods. Decreased palmitate oxidation of the HFD-fed $\mathrm{Mcd}^{-/-}$mice improved insulin sensitivity with reduced incomplete fatty acid oxidation [3], supporting the hypothesis that increased incomplete fatty acid oxidation is one of the potential mechanisms of HFD-induced insulin resistance. Unexpectedly, our experiment using the same protocol did not detectedany difference of incomplete fatty acid oxidation in isolated mitochondria between $C p t 1 b^{+/-}$and WT muscle under a HFD condition. While the reasons for this discrepancy remain incompletely understood, we suspect that blocking the entry of long chain fatty acids into the mitochondria of $C p t 1 b^{+/-}$muscle may not be robust enough to cause incomplete fatty acid oxidation, yet is sufficient to repress fatty acid oxidation and upregulating glucose oxidation in skeletal muscle. Therefore, it is probable that partial CPT1b repression protects mice from HFD-induced insulin resistance by attenuating HFDinduced upregulation of fatty acid oxidation and upregulating glucose oxidation in skeletal muscle. Since it has been extensively proposed that intramyocellular lipid accumulation may be one of the main causes of insulin resistance, a potential detrimental effect after prolonged intramyocellular lipid accumulation is still likely. It is possible that the suppressed FAO in $\mathrm{Cpt} 1 \mathrm{~b}^{+/-}$mice did not reach the threshold that leads to the mismatch of lipid supply and degradation and subsequent intramyocellular lipid accumulation.

The insulin sensitizing effect of CPT1b deficiency is also evident by the remarkable difference in the insulin-stimulated phosphorylation of Ser302 of insulin receptor substrate 1 (IRS1) in the skeletal muscle. Ser302 in rat/mouse IRS-1 (corresponding to Ser307 of human IRS-1) is one of the molecular mechanisms proposed as an indicator of cellular energy status underpinning the development of insulin resistance $[39,40]$. Transgenic mice with muscle-specific IRS-1 serine to alanine are protected from fat-induced insulin resistance in skeletal muscle [41]. Since the phosphorylation of IRS1 at Ser302 is substantially induced by hyperinsulinemia [40], the lower insulin level (Table 1) may also contribute to the less phosphorylation of IRS1 at Ser302 in

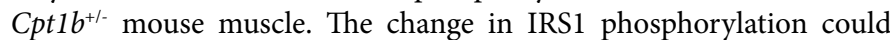
only be detected in skeletal muscle but not in other tissues, supporting that skeletal muscle is the main tissue responsible for the improved insulin sensitivity in $C p t 1 b^{+/-}$mice under a HFD condition. Therefore, our results support that $\mathrm{CPT} 1 \mathrm{~b}$ repression sensitizes insulin action via increased glucose oxidation and insulin signaling in skeletal muscle. It is not clear why the insulin signal transduction in GWAT was suppressed at this point, but it appears that was not sufficient to offset the insulin sensitizing effect of partial CPT1b repression in skeletal muscle.

The insulin sensitizing effect of partial CPT1b deficiency appears to be unrelated to a decrease of relative body fat, since the fat mass was not correlated with mouse genotype assessed by ANCOVA. Despite the matched fat/lean mass ratio between the $C p t 1 b^{+/-}$and WT mice, serum level of leptin was markedly higher in WT than in $C p t 1 b^{+/-}$mice (Table 1). It is plausible that the greater total body fat in the HFD-fed WT mice may contribute to the higher serum level of leptin. It is intriguing that the $\mathrm{Cpt}_{1 \mathrm{~b}^{+/}}$mice showed a slower growth rate than their WT counterparts in response to HFD with proportional decreases of fat and lean mass. Albeit the exact mechanism underpinning this phenomenon is not clear, it is plausible that a potentially less energy production rate from glucose oxidation may play certain roles. Because of the lack of neurohumoral responses, the ex vivo oxidation assays using isolated muscle, isolated mitochondria, and homogenate may not reflect what exactly happens under the in vivo condition. This may explain why the CPT1b deficient mice showed repressed fatty acid oxidation and increased glucose oxidation in skeletal muscle, yet without major shift in whole body metabolism $[42,43]$.

Interestingly, the effects of CPT1 deficiencies on growth and insulin resistance are subtype-dependent. One study found that $C p t 1 c^{+-}$mice are smaller than WT mice even under a CHD condition and they are susceptible to insulin resistance, obesity, and hepatosteatosis under a HFD condition [44]. Previously we reported that the Cpt1 $a^{+/-}$mice were resistant against HFD-induced insulin resistance, but showed hepatosteatosis with increased hepatic CPT1b expression [45]. We also found that $\mathrm{Cpt} 1 \mathrm{~b}^{+/-}$mice showed $40 \%$ decreased CPT1 activity in muscle and there were no significant changes in body weight, tissue histology, serum free fatty acid [16], and normal cardiac function and heart weight compared to WT littermates [46]. In this study we found that $C p t 1 b^{+/-}$mice showed no difference in glucose and insulin tolerance under a CHD condition at least up to 32 weeks of age. Insulin sensitivity of $\mathrm{Cpt} 1 \mathrm{~b}^{+/-}$mice is well maintained under a HFD condition. Therefore, it appears that CPT1b is a better specific CPT1 target for the treatment of insulin resistance.

Taken together, the current investigation confirmed the beneficial effects of CPT1b repression on diet-induced insulin resistance. Further studies will be required to identify CPT1b-specific inhibitors, and to confirm the effectiveness and safety of prolonged inhibition of CPT1b in animals and in patients with insulin resistance and type II diabetes.

\section{Acknowledgements}

The technical services of the UAB Metabolism Core Laboratory were supported by grants P30DK56336, UL 1RR025777, and P60DK079626. Small Animal Phenotyping Core was supported by UAB Diabetes Research Center P60DK079626 and the UAB Nutrition Obesity Research Center P30DK056336. The Targeted Metabolomics and Proteomics Laboratory were supported partly by P30DK079337 and P30AR50948. The opinions expressed in this article are not those of the NIH or any other organization with which the authors are affiliated.

\section{Grants}

This work was supported by grants from National Institutes of Health (1RO1 
Citation: Kim T, He L, Johnson MS, Li Y, Zeng L, et al. (2014) Carnitine Palmitoyltransferase 1b Deficiency Protects Mice from Diet-Induced Insulin Resistance. J Diabetes Metab 5: 361 doi:10.4172/2155-6156.1000361

HL085499 and 1RO1 HL084456 to Dr. Yang, 1RO1 RR02599 to Dr. Wood, and T32 HL007457 to Dr. Kim).

\section{Disclosures}

The authors declare that there are no conflicts of interest and financial associated with this study and the report presented here. The statements and opinions contained in articles are solely those of the individual authors and contributors and not of the Journal of Diabetes and Metabolism.

\section{References}

1. Hegarty BD, Cooney GJ, Kraegen EW, Furler SM (2002) Increased efficiency of fatty acid uptake contributes to lipid accumulation in skeletal muscle of high fat-fed insulin-resistant rats. Diabetes 51: 1477-1484

2. Schreurs M, Kuipers F, van der Leij FR (2010) Regulatory enzymes of mitochondrial beta-oxidation as targets for treatment of the metabolic syndrome. Obes Rev 11: 380-388.

3. Koves TR, Ussher JR, Noland RC, Slentz D, Mosedale M, et al. (2008) Mitochondrial overload and incomplete fatty acid oxidation contribute to skeleta muscle insulin resistance. Cell Metab 7: 45-56.

4. Keung W, Ussher JR, Jaswal JS, Raubenheimer M, Lam VH, et al. (2013) Inhibition of carnitine palmitoyltransferase-1 activity alleviates insulin resistance in diet-induced obese mice. Diabetes 62: 711-720.

5. Collier GR, Traianedes K, Macaulay SL, O'Dea K (1993) Effect of fatty acid oxidation inhibition on glucose metabolism in diabetic rats. Horm Metab Res 25: 9-12.

6. Conti R, Mannucci E, Pessotto P, Tassoni E, Carminati P, et al. (2011) Selective reversible inhibition of liver carnitine palmitoyl-transferase 1 by teglicar reduces gluconeogenesis and improves glucose homeostasis. Diabetes 60: 644-651.

7. Timmers S, Nabben M, Bosma M, van Bree B, Lenaers E, et al. (2012) Augmenting muscle diacylglycerol and triacylglycerol content by blocking fatty acid oxidation does not impede insulin sensitivity. Proc Natl Acad Sci U S A 109: 11711-11716.

8. Ratheiser K, Schneeweiss B, WaldhäusI W, Fasching P, Korn A, et al. (1991) Inhibition by etomoxir of carnitine palmitoyltransferase I reduces hepatic glucose production and plasma lipids in non-insulin-dependent diabetes mellitus. Metabolism 40: 1185-1190.

9. Hübinger A, Knode O, Susanto F, Reinauer H, Gries FA (1997) Effects of the carnitine-acyltransferase inhibitor etomoxir on insulin sensitivity, energy expenditure and substrate oxidation in NIDDM. Horm Metab Res 29: 436-439.

10. Rufer AC, Thoma R, Hennig M (2009) Structural insight into function and regulation of carnitine palmitoyltransferase. Cell Mol Life Sci 66: 2489-2501.

11. Turner N, Bruce CR, Beale SM, Hoehn KL, So T, et al. (2007) Excess lipid availability increases mitochondrial fatty acid oxidative capacity in muscle: evidence against a role for reduced fatty acid oxidation in lipid-induced insulin resistance in rodents. Diabetes 56: 2085-2092.

12. Jans A, Sparks LM, van Hees AM, Gjelstad IM, Tierney AC, et al. (2011) Transcriptional metabolic inflexibility in skeletal muscle among individuals with increasing insulin resistance. Obesity (Silver Spring) 19: 2158-2166.

13. Schmitz FJ, Rösen P, Reinauer H (1995) Improvement of myocardial function and metabolism in diabetic rats by the carnitine palmitoyl transferase inhibitor Etomoxir. Horm Metab Res 27: 515-522.

14. Swislocki A, Eason T (1994) Glucose tolerance and blood pressure are improved in the spontaneously hypertensive rat by ethyl-2-(6-(4-chlorophenoxy)-hexyl) oxirane-2-carboxylate (etomoxir), an inhibitor of fatty acid oxidation. Am J Hypertens 7: 739-744.

15. Bruce CR, Hoy AJ, Turner N, Watt MJ, Allen TL, et al. (2009) Overexpression of carnitine palmitoyltransferase-1 in skeletal muscle is sufficient to enhance fatty acid oxidation and improve high-fat diet-induced insulin resistance. Diabetes 58: $550-558$

16. Ji S, You Y, Kerner J, Hoppel CL, Schoeb TR, et al. (2008) Homozygous carnitine palmitoyltransferase $1 \mathrm{~b}$ (muscle isoform) deficiency is lethal in the mouse. Mol Genet Metab 93: 314-322.

17. Zong H, Wang CC, Vaitheesvaran B, Kurland IJ, Hong W, et al. (2011) Enhanced energy expenditure, glucose utilization, and insulin sensitivity in VAMP8 null mice. Diabetes 60: 30-38.

18. Choi CS, Savage DB, Abu-Elheiga L, Liu ZX, Kim S, et al. (2007) Continuous fat oxidation in acetyl-CoA carboxylase 2 knockout mice increases total energy expenditure, reduces fat mass, and improves insulin sensitivity. Proc Natl Acad Sci U S A 104: 16480-16485.

19. Ayala JE, Bracy DP, McGuinness OP, Wasserman DH (2006) Considerations in the design of hyperinsulinemic-euglycemic clamps in the conscious mouse. Diabetes 55: 390-397.

20. Youn JH, Kim JK, Buchanan TA (1994) Time courses of changes in hepatic and skeletal muscle insulin action and GLUT4 protein in skeletal muscle after STZ injection. Diabetes 43: 564-571.

21. Primassin S, Tucci S, Herebian D, Seibt A, Hoffmann L, et al. (2010) Pre-exercise medium-chain triglyceride application prevents acylcarnitine accumulation in skeletal muscle from very-long-chain acyl-CoA-dehydrogenase-deficient mice. $\mathrm{J}$ Inherit Metab Dis 33: 237-246.

22. Bligh EG, Dyer WJ (1959)A rapid method of total lipid extraction and purification Can J Biochem Physiol 37: 911-917.

23. ter Veld F, Primassin S, Hoffmann L, Mayatepek E, Spiekerkoetter U (2009) Corresponding increase in long-chain acyl-CoA and acylcarnitine after exercise in muscle from VLCAD mice. J Lipid Res 50: 1556-1562.

24. Kim KH, Jeong YT, Oh H, Kim SH, Cho JM, et al. (2013) Autophagy deficiency leads to protection from obesity and insulin resistance by inducing Fgf21 as a mitokine. Nat Med 19: 83-92.

25. Jones AS, Johnson MS, Nagy TR (2009) Validation of quantitative magnetic resonance for the determination of body composition of mice. Int $\mathrm{J}$ Body Compos Res 7: 67-72.

6. Bray MS, Ratcliffe WF, Grenett MH, Brewer RA, Gamble KL, et al. (2013) Quantitative analysis of light-phase restricted feeding reveals metabolic dyssynchrony in mice. Int J Obes (Lond) 37: 843-852.

27. Li X, Johnson MS, Smith DL Jr, Li Y, Kesterson RA, et al. (2013) Effects of risperidone on energy balance in female C57BL/6J mice. Obesity (Silver Spring) 21: 1850-1857

28. Foley JE (1992) Rationale and application of fatty acid oxidation inhibitors in treatment of diabetes mellitus. Diabetes Care 15: 773-784.

29. Wolf HP (1992) Possible new therapeutic approach in diabetes mellitus by inhibition of carnitine palmitoyltransferase 1 (CPT1). Horm Metab Res Suppl 26: $62-67$

30. Giannessi F, Chiodi P, Marzi M, Minetti P, Pessotto P, et al. (2001) Reversible carnitine palmitoyltransferase inhibitors with broad chemical diversity as potential antidiabetic agents. J Med Chem 44: 2383-2386.

31. Dobbins RL, Szczepaniak LS, Bentley B, Esser V, Myhill J, et al. (2001) Prolonged inhibition of muscle carnitine palmitoyltransferase-1 promotes intramyocellular lipid accumulation and insulin resistance in rats. Diabetes 50 : 123-130.

32. Rupp H, Rupp TP, Maisch B (2005) Fatty acid oxidation inhibition with PPARalpha activation (FOXIB/PPARalpha) for normalizing gene expression in heart failure? Cardiovasc Res 66: 423-426.

33. Weis BC, Cowan AT, Brown N, Foster DW, McGarry JD (1994) Use of a selective inhibitor of liver carnitine palmitoyltransferase I (CPT I) allows quantification of its contribution to total CPT I activity in rat heart. Evidence that the dominan cardiac CPT I isoform is identical to the skeletal muscle enzyme. J Biol Chem 269: $26443-26448$

34. Stephens TW, Higgins AJ, Cook GA, Harris RA (1985) Two mechanisms produce tissue-specific inhibition of fatty acid oxidation by oxfenicine. Biochem J 227: 651-660.

35. Paumen MB, Ishida Y, Muramatsu M, Yamamoto M, Honjo T (1997) Inhibition of carnitine palmitoyltransferase I augments sphingolipid synthesis and palmitateinduced apoptosis. J Biol Chem 272: 3324-3329.

36. Hulver MW, Berggren JR, Cortright RN, Dudek RW, Thompson RP, et al. (2003) Skeletal muscle lipid metabolism with obesity. Am J Physiol Endocrinol Metab 284: E741-747.

37. Kim JY, Hickner RC, Cortright RL, Dohm GL, Houmard JA (2000) Lipid oxidation is reduced in obese human skeletal muscle. Am J Physiol Endocrinol Metab 279: E1039-1044.

38. Phielix E, Mensink M (2008) Type 2 diabetes mellitus and skeletal muscle metabolic function. Physiol Behav 94: 252-258. 
Citation: Kim T, He L, Johnson MS, Li Y, Zeng L, et al. (2014) Carnitine Palmitoyltransferase 1b Deficiency Protects Mice from Diet-Induced Insulin Resistance. J Diabetes Metab 5: 361 doi:10.4172/2155-6156.1000361

Page 11 of 11

39. Werner ED, Lee J, Hansen L, Yuan M, Shoelson SE (2004) Insulin resistance due to phosphorylation of insulin receptor substrate-1 at serine 302 . J Biol Chem 279: 35298-35305.

40. Giraud J, Leshan R, Lee YH, White MF (2004) Nutrient-dependent and insulinstimulated phosphorylation of insulin receptor substrate-1 on serine 302 correlates with increased insulin signaling. J Biol Chem 279: 3447-3454.

41. Morino K, Neschen S, Bilz S, Sono S, Tsirigotis D, et al. (2008) Musclespecific IRS-1 Ser->Ala transgenic mice are protected from fat-induced insulin resistance in skeletal muscle. Diabetes 57: 2644-2651.

42. Muoio DM, Neufer PD (2012) Lipid-induced mitochondrial stress and insulin action in muscle. Cell Metab 15: 595-605.

43. Hoehn KL, Turner N, Swarbrick MM, Wilks D, Preston E, et al. (2010) Acute or chronic upregulation of mitochondrial fatty acid oxidation has no net effect on whole-body energy expenditure or adiposity. Cell Metab 11: 70-76.

44. Wolfgang MJ, Kurama T, Dai Y, Suwa A, Asaumi M, et al. (2006) The brainspecific carnitine palmitoyltransferase- $1 \mathrm{c}$ regulates energy homeostasis. Proc Natl Acad Sci U S A 103: 7282-7287.

45. Nyman LR, Tian L, Hamm DA, Schoeb TR, Gower BA, et al. (2011) Long term effects of high fat or high carbohydrate diets on glucose tolerance in mice with heterozygous carnitine palmitoyltransferase-1a (CPT-1a) deficiency: Diet influences on CPT1a deficient mice. Nutr Diabetes 1: e14.

46. He L, Kim T, Long Q, Liu J, Wang P, et al. (2012) Carnitine palmitoyltransferase$1 \mathrm{~b}$ deficiency aggravates pressure overload-induced cardiac hypertrophy caused by lipotoxicity. Circulation 126: 1705-1716. 